\title{
Método conservativo de diferencias finitas de alto orden para una clase de sistemas de Schrödinger no lineales
}

\author{
A. Aguilera ${ }^{a, *}$, P. Castillo ${ }^{a, * *}$ y S. Gómez ${ }^{b}$ \\ ${ }^{a}$ Department of Mathematical Sciences, University of Puerto Rico, \\ Call Box 9000, Mayagüez, Puerto Rico 00681-9018. \\ e-mail:*axi.aguilera@upr.edu, ${ }^{* *}$ paul.castillo@upr.edu \\ ${ }^{b}$ Dipartamento di Matematica "F. Casorati", Università di Pavia, \\ Via Ferrata 5, 27100 Pavia, Italy. \\ e-mail:sergio.gomez01@universitadipavia.it
}

Received 16 August 2021; accepted 22 September 2021

Se presenta un método en tiempo para aproximar la solución de una clase de sistemas de ecuaciones no lineales de Schrödinger, el cual conserva la potencia de cada componente y el Hamiltoniano del sistema de manera exacta. Para la discretización espacial se consideran fórmulas explícitas y compactas de diferencias finitas, ambas de cuarto y sexto orden; sin embargo, fórmulas de mayor orden también podrían ser utilizadas. La técnica para avanzar en tiempo se basa en una modificación del esquema conservativo de Crank-Nicolson, la cual se aplica de manera secuencial a cada una de las componentes del campo vectorial. La conservación de los invariantes discretos y el orden de convergencia del método se validan por medio de una serie de experimentos numéricos utilizando diferentes potenciales no lineales.

Descriptores: Sistemas de Schrödinger no lineales; conservación de potencia y energía; métodos conservativos; diferencias finitas de alto orden.

A time method to approximate the solution of a class of nonlinear Schrödinger systems, which preserves the power of each component and the Hamiltonian of the system exactly, is presented. Spatial discretizations based on fourth- and sixth-order explicit and compact finite difference formulas are considered; however, higher order formulas could also be used. The time advancing technique is based on a modification of a conservative Crank-Nicolson scheme, which is applied sequentially to each of the components of the vector field. Conservation of discrete invariants and order of convergence of the method are validated by means of a series of numerical experiments using different nonlinear potentials.

Keywords: Nonlinear Schrödinger systems; power and energy conservation; conservative methods; high order finite differences.

DOI: https://doi.org/10.31349/RevMexFisE.19.010205

\section{Introducción}

La ecuación de Schrödinger no lineal es una de las más importantes en la mecánica cuántica. Esta modela diversos fenómenos físicos no lineales como, propagación de haces ópticos con diferentes tipos de no linealidades, condensados de Bose-Einsten, redes de transmisión no lineal, física de plasma, entre otros [1-4]. Debido a la variedad de aplicaciones físicas, sistemas de ecuaciones de Schrödinger no lineales fuertemente acopladas, han sido estudiados desde finales de la década del sesenta y se han convertido en interés de estudio para muchos investigadores. Por ejemplo, en Ref. [5] se derivó un modelo para varios paquetes de onda que interactúan en un sistema conservativo, en Ref. [6] se introdujo el bien conocido sistema de Manakov que modela solitones electromagnéticos en fibra birrefrigente, en Ref. [7] se estudian sistemas que describen diferentes tipos de solitones ópticos y en Ref. [8] se estudian numéricamente problemas que surgen en la dinámica de láser ultrarrápida.

En este trabajo se desea aproximar de manera precisa la solución de este tipo de sistemas, reproduciendo numéricamente algunas propiedades físicas, principalmente, la conservación de ciertos invariantes. Las componentes, $j=$
$1, \ldots, J, J>1$, del campo complejo $\Psi=\left(\psi_{1}, \ldots, \psi_{J}\right)$ : $\Omega=(a, b) \longrightarrow \mathbb{C}^{J}$, satisfacen el siguiente sistema de ecuaciones no lineales, en el dominio espacio-tiempo $(a, b) \times$ $(0, T]$,

$$
i \frac{\partial \psi_{j}}{\partial t}=-\sigma_{j} \frac{\partial^{2} \psi_{j}}{\partial^{2} x}-f_{j}\left(\left|\psi_{1}\right|^{2}, \ldots,\left|\psi_{J}\right|^{2}\right) \psi_{j},
$$

con condiciones periódicas. Se asume que para cada $j=$ $1, \ldots, J$ la constante de dispersión $\sigma_{j} \in \mathbb{R}$; y la existencia de una función $F: \mathbb{R}^{J} \longrightarrow \mathbb{R}$ tal que $\nabla F=\left(f_{j}\right)$.

Para poner el presente trabajo en contexto y sin pretender hacer una revisión de literatura exhaustiva, repasamos brevemente algunos trabajos relacionados exclusivamente con métodos numéricos diseñados para sistemas de Schrödinger no lineales (1). Si bien es cierto que métodos como el de elemento finito clásico, elemento finito discontinuo, métodos espectrales y técnicas basadas en B-splines han sido ampliamente utilizados para la discretización del operador diferencial de segundo orden, debido a la sencillez de su formulación e implementación, el método de diferencias finitas es uno de los preferidos. Su derivación requiere el conocimiento de pocas herramientas matemáticas, principalmente el Teorema de Taylor. La fórmula de diferenciación central clásica 
para la segunda derivada ha sido empleada en [9-17]. Sin embargo, como es bien sabido, esta es únicamente de segundo orden. Para obtener mayor precisión, aproximaciones de alto orden en espacio deben ser empleadas. Por ejemplo, aproximaciones de cuarto y sexto orden en espacio han sido utilizadas por diversos autores [18-22]. Estas se basan en fórmulas de diferencias finitas centrales clásicas y fórmulas compactas de alto orden cuyo objetivo principal es la de obtener una matriz de discretización con el menor esténcil posible; por ejemplo, para cuarto orden es posible generar fórmulas cuya matriz es tridiagonal; a diferencia de la fórmula clásica de mismo orden, cuya matriz correspondiente es pentadiagonal.

El sistema modelo (1) satisface varias leyes de conservación, siendo la conservación de la potencia de cada componente (2a) y del Hamiltoniano (energía) del sistema (2b), de las más relevantes. Estos invariantes se definen formalmente de la siguiente manera

$$
\begin{aligned}
\mathcal{P}_{j}(t):= & \int_{a}^{b}\left|\psi_{j}(x, t)\right|^{2} \mathrm{dx}, \quad j=1, \ldots, J \\
\mathcal{H}(t):= & \frac{1}{2}\left(\sum_{j=1}^{J} \int_{a}^{b} \sigma_{j}\left|\frac{\partial \psi_{j}}{\partial x}(x, t)\right|^{2} \mathrm{dx}\right. \\
& \left.-\int_{a}^{b} F\left(|\Psi(x, t)|^{2}\right) \mathrm{dx}\right),
\end{aligned}
$$

donde $|\Psi|^{2}:=\left(\left|\psi_{1}\right|^{2}, \ldots,\left|\psi_{J}\right|^{2}\right)$.

La conservación de los análogos discretos de estos invariantes es de vital importancia en el diseño de esquemas numéricos para la aproximación de la solución de sistemas de Schrödinger no lineales, no solo por tratarse de cantidades de interés físico, sino que además está íntimamente relacionada con la estabilidad y convergencia del método. Además los esquemas conservativos resultan más apropiados para simulaciones en largos períodos de tiempo.

Motivados por el concepto, introducido por Bridges y Reich [23], de método multi-simpléctico; es decir un esquema que preserva el análogo discreto de la simplecticidad de una ecuación Hamiltoniana en derivadas parciales, diversos esquemas han sido propuestos. La discretización de punto medio en tiempo y en espacio, conocida como esquema de Preissman fue considerado, por primera vez, por Sun y Qin [24], para un sistema de Schrödinger no lineal de 2 componentes y luego por Aydin [25] para un sistema de 3 componentes con potencial cúbico. Esquemas conservativos semi explícitos derivados del método Euler-Box fueron adaptados por Cai [16] a sistemas de dos componentes. Esquemas basados en una descomposición multi-simpléctica lineal y un operador no lineal han sido propuestos por diversos autores [26-29]. La combinación de fórmulas compactas de diferencias finitas de cuarto orden con los esquemas de avance en tiempo, conocidos en inglés como "Average Vec- tor Field" y "Hamiltonian Boundary Value", fueron recientemente analizados por Kong et al., [30] y Son et al., [31], respectivamente. Esquemas numéricos que conservan localmente la potencia y el momentum, fueron desarrollados por Cai et al., [32].

La dificultad del problema, desde un punto de vista numérico, estriba en la conservación de ambos invariantes discretos de manera eficiente. En [33], Castillo y Gómez, utilizando discretizaciones espaciales de alto orden basadas en el método "Local Discontinuous Galerkin" (LDG), presentaron un esquema que conserva la potencia de cada componente y el Hamiltoniano del sistema en precisión de máquina. Un análisis de convergencia fue presentado recientemente por Aguilera, Castillo y Gómez [34], para un sistema de Schrödinger con potencial cúbico, utilizando diferencias centrales para la aproximación de la segunda derivada. Específicamente, se demostró que para este problema nuestro método básico converge de orden $\tau+h^{2}$; donde $\tau$ y $h$ son los parámetros de discretización temporal y espacial, respectivamente. Además se desarrollaron métodos de orden $\tau^{2}+h^{2} \mathrm{y}$ $\tau^{4}+h^{2}$ basados en la composición de esquemas temporales.

Asumiendo que la solución del problema posee suficiente regularidad, una forma eficiente de obtener mayor precisión consiste en utilizar aproximaciones de alto orden en mallas no muy finas. Esto fue claramente evidenciado en los experimentos numéricos presentados por Castillo y Gómez [33, 35] para sistemas de una y varias componentes, usando aproximaciones polinomiales de alto orden. Motivados por estas ideas, en este trabajo se extiende el esquema numérico presentado en [34] a discretizaciones de diferencias finitas de alto orden. Además ilustramos numéricamente la conservación de ambos invariantes para diferentes potenciales, como por ejemplo en el caso de saturación. El esquema propuesto posee las siguientes propiedades:

a) La conservación de los análogos discretos de la potencia y del Hamiltoniano es garantizada utilizando un barrido de tipo Gauss-Seidel no lineal en las componentes del campo. Como consecuencia, el sistema no lineal global se descompone en sistemas no lineales de menor tamaño.

b) Aproximaciones de alta precisión son obtenidas combinando fórmulas de diferencias finitas de alto orden para la discretización espacial y técnicas de composición de esquemas en tiempo.

c) La discretización del término no lineal se basa en la estrategia presentada por Delfour, Fortin y Payré [9], denotada por DFP, la cual es independiente de la fórmula de diferencias finitas utilizada para el operador diferencial de segundo orden.

El artículo está estructurado de la siguiente manera: en la Sec. 2 se presenta un breve repaso de la derivación de fórmulas de diferencias finitas de alto orden. En particular, se discute una técnica para generar fórmulas implícitas de alto orden. La idea central del esquema en tiempo es presentada en 
la Sec. 3 y las propiedades de conservación son analizadas en la Sec. 4. Algunos detalles generales sobre implementación son discutidos en la Sec. 5 y la validación numérica del esquema se ilustra en la Sec. 6 .

\section{Diferencias finitas de alto orden}

Fórmulas de diferencias finitas para aproximar las derivadas de una función se obtienen, por lo general, manipulando directamente desarrollos de Taylor. Es bien sabido que dichas fórmulas pierden localidad a medida que se aumenta el orden de precisión, lo cual tiene un impacto negativo en el rendimiento de un esquema numérico debido al aumento en el costo computacional que conlleva la evaluación del operador diferencial discreto en cada paso de tiempo. Con el propósito de solventar este problema, diversas técnicas para generar aproximaciones más compactas han sido desarrolladas. En esta sección, se repasa brevemente un marco general para generar aproximaciones de derivadas mediante fórmulas de diferencias finitas de alto orden, presentado por Lele [36]. Nos enfocaremos, particularmente, en aquellas diseñadas para la segunda derivada. A diferencia de la técnica tradicional, el procedimiento es de carácter global, es decir, involucra de manera implícita todos los nodos de una malla del dominio $\Omega=(a, b)$.

Sea $\mathcal{T}_{h}$ una discretización de $\Omega$ por medio de una malla de $M+1$ puntos uniformemente espaciados

$$
\mathcal{T}_{h}=\left\{a=x_{0}<\ldots<x_{M}=b\right\},
$$

donde $h=(b-a) / M=x_{j+1}-x_{j}$ para $j=0, \ldots M-1$. Los operadores discretos clásicos para la segunda derivada se denotan por

$$
\begin{aligned}
\delta_{h}^{0}\left(\psi_{i}\right) & :=\frac{1}{h^{2}}\left(\psi\left(x_{i-1}\right)-2 \psi\left(x_{i}\right)+\psi\left(x_{i+1}\right)\right) \\
\delta_{h}^{1}\left(\psi_{i}\right) & :=\frac{1}{4 h^{2}}\left(\psi\left(x_{i-2}\right)-2 \psi\left(x_{i}\right)+\psi\left(x_{i+2}\right)\right)
\end{aligned}
$$

Puesto que se consideran condiciones de borde periódicas, el nodo $x_{0}$ se identifica con el nodo $x_{M}$; por lo tanto, las matrices $\Delta_{0}$ y $\Delta_{1}$ asociadas a los operadores discretos $\delta_{h}^{0}$ y $\delta_{h}^{1}$, respectivamente, son simétricas y circulantes. Utilizando la representación compacta de una matriz circulante

$\operatorname{Circ}\left(c_{0}, \ldots, c_{M-1}\right):=\left(\begin{array}{ccccc}c_{0} & c_{1} & \ldots & c_{M-2} & c_{M-1} \\ c_{M-1} & c_{0} & c_{1} & \ddots & c_{M-2} \\ \ldots & \ddots & \ddots & \ddots & \ldots \\ c_{2} & \ddots & \ddots & c_{0} & c_{1} \\ c_{1} & c_{2} & \ldots & c_{M-1} & c_{0}\end{array}\right)$,

se tiene

$$
\begin{aligned}
\Delta_{0} & :=\frac{1}{h^{2}} \operatorname{Circ}\left(\begin{array}{llllllll}
-2 & 1 & 0 & 0 & \ldots & 0 & 0 & 1
\end{array}\right), \\
\Delta_{1} & :=\frac{1}{4 h^{2}} \operatorname{Circ}\left(\begin{array}{llllllll}
-2 & 0 & 1 & 0 & \ldots & 0 & 1 & 0
\end{array}\right) .
\end{aligned}
$$

Denotando por $\delta_{i}$ la aproximación de $\psi^{\prime \prime}\left(x_{i}\right)$, se buscan los coeficientes $\alpha_{j}, j=1, \ldots, L$ y $a, b$ de tal manera que

$$
\sum_{j=0}^{L} \alpha_{j} \delta_{i \pm j}=a \delta_{h}^{0} \psi\left(x_{i}\right)+b \delta_{h}^{1} \psi\left(x_{i}\right), \quad \text { con } \alpha_{0}=1 .
$$

En notación matricial, este sistema se reescribe como

$$
A\left(\delta_{i}\right)=D\left(\psi\left(x_{i}\right)\right), \quad \text { donde } D=a \Delta_{0}+b \Delta_{1} .
$$

$A$ es una matriz simétrica circulante, con $L+1$ diagonales, en la cual todos los elementos de su diagonal principal toman el valor de 1 ,

$$
A:=\operatorname{Circ}\left(1, \alpha_{1}, \ldots, \alpha_{L}, 0, \ldots, 0, \alpha_{L}, \ldots, \alpha_{1}\right)
$$

Cuando $\alpha_{j}=0$ para $j=1, \ldots, L, A$ se reduce a la matriz identidad $I_{M}$, y se dice que la fórmula es explícita. Por el contrario, si $\alpha_{j} \neq 0$ para algún $j \in\{1, \ldots, L\}$, la fórmula se dice ser implícita. Si bien es cierto que las aproximaciones $\delta_{i}$ pueden obtenerse calculando la matriz $\Delta_{h}:=A^{-1} D$, esta requiere del conocimiento explícito de $A^{-1}$ el cual no se tiene en la práctica.

Para un $L$ fijo, los coeficientes deben satisfacer ciertas condiciones de orden óptimo, las cuales se obtienen a partir del desarrollo de Taylor del error de truncamiento $T_{i}$, definido por

$$
T_{i}:=\sum_{j=0}^{L} \alpha_{j} \psi^{\prime \prime}\left(x_{i \pm j}\right)-\left(a \delta_{h}^{0} \psi\left(x_{i}\right)+b \delta_{h}^{1} \psi\left(x_{i}\right)\right) .
$$

En este trabajo, se asumirá $L=2$ por lo que las condiciones de orden se reducen a tres:

$$
\begin{aligned}
a+b & =1+2 \alpha_{1}+2 \alpha_{2} \\
a+4 b & =\frac{4 !}{2 !}\left(\alpha_{1}+4 \alpha_{2}\right) \\
a+16 b & =\frac{6 !}{4 !}\left(\alpha_{1}+16 \alpha_{2}\right)
\end{aligned}
$$

Para una fórmula de orden 4, las condiciones 1) y 2) deben cumplirse, lo cual resulta en una familia descrita por 2 parámetros. Consideramos las siguientes fórmulas de orden 4

$$
\begin{aligned}
& -\alpha_{1}=\alpha_{2}=0, \quad a=\frac{4}{3}, b=-\frac{1}{3} . \\
& \text { - } \quad \alpha_{1}=\frac{1}{10}, \alpha_{2}=0, \quad a=\frac{12}{10}, b=0 .
\end{aligned}
$$

La segunda fórmula es implícita; y a diferencia de la primera, es la única fórmula compacta de cuarto orden, en la cual ambas matrices $A$ y $D$ son tridiagonales; lo que es extremadamente ventajoso desde el punto de vista computacional.

Para obtener fórmulas de orden 6, las tres condiciones deben cumplirse por lo que se obtiene una familia de fórmulas que depende de un solo parámetro. Desafortunadamente, 
las condiciones de orden no permiten construir una fórmula para la cual ambas matrices $A$ y $D$ sean tridiagonales. Por ejemplo, en la siguiente fórmula compacta de orden $6, A$ es tridiagonal, mientras que $D$ es pentadiagonal

$$
\text { - } \quad \alpha_{1}=\frac{2}{11}, \alpha_{2}=0, \quad a=\frac{12}{11}, b=\frac{3}{11} .
$$

La simetría del operador diferencial discreto es de vital importancia para garantizar la conservación de los invariantes discretos. A continuación probamos esta propiedad.

Proposición 1 Asumiendo que A es una matriz no singular, entonces $\Delta_{h}$ es simétrica.

Prueba. Puesto que el espacio de las matrices circulantes es un anillo conmutativo [37], $A$ conmuta con las matrices $\Delta_{0}$ y $\Delta_{1} ;$ y por lo tanto con $D$. Luego,

$$
A^{-1} D A=A^{-1} A D=D \quad \Longleftrightarrow \quad A^{-1} D=D A^{-1} .
$$

Sea $A^{*}$ la adjunta de $A$, utilizando el resultado anterior y la simetría de $A^{-1}$ y $D$ se tiene

$$
\Delta_{h}^{*}=\left(A^{-1} D\right)^{*}=D^{*}\left(A^{-1}\right)^{*}=D A^{-1}=\Delta_{h} .
$$

\section{Discretización en tiempo}

Para garantizar la conservación del Hamiltoniano a nivel discreto es necesario tratar el término no lineal de manera especial. En la Ref. [33], Castillo y Gómez extienden a sistemas de tipo (1) la técnica (DFP) presentada por Delfour, Fortin y Payré [9] para una ecuación cúbica de Schrödinger; la cual a su vez fue propuesta originalmente por Strauss y Vazquez [38] para una ecuación no lineal de Klein-Gordon. Sea $\tau$ el incremento en tiempo, el cual se asumirá constante. En cada tiempo discreto $t_{n}=n \tau$ y nodo $x_{m} \in \mathcal{T}_{h}$, la técnica DFP utiliza la expresión del potencial no lineal $F(\cdot)$ en la discretización del término no lineal. Esta se define de la siguiente manera

$$
\mathcal{N}_{h}\left(\psi_{m}^{n}\right):=\frac{F\left(\left|\psi_{m}^{n+1}\right|^{2}\right)-F\left(\left|\psi_{m}^{n}\right|^{2}\right)}{\left|\psi_{m}^{n+1}\right|^{2}-\left|\psi_{m}^{n}\right|^{2}},
$$

y puede interpretarse como una aproximación de $\frac{\partial F}{\partial \xi}\left(\left|\Psi^{n}\right|^{2}\right):=f\left(\left|\Psi^{n}\right|^{2}\right)$. Para potenciales de tipo polinomial, el operador discreto $\mathcal{N}_{h}(\cdot)$ puede simplificarse con el fin de evitar el problema numérico conocido como cancelación catastrófica, el cual puede ocurrir cuando el denominador es relativamente muy pequeño en precisión de máquina.

La técnica DFP también ha sido utilizada exitosamente en combinación con métodos de elemento finito. En la Ref. [39] Sanz-Serna presentó un análisis de convergencia para el método de elemento finito clásico aplicado a una ecuación de Schrödinger de término no lineal general; y recientemente, el método LDG fue considerado por Castillo y Gómez [40, 41] como discretización espacial de alto orden, para ecuaciones no lineales de Schrödinger con operadores diferenciales fraccionarios.

Desafortunadamente, la aproximación dada por la expresión (10) no puede ser aplicada directamente en el caso de sistemas $(J>1)$. Sin embargo, si en cada paso de tiempo, el problema global se descompone de manera secuencial en sub-problemas asociados a cada una de las componentes del campo, se obtiene un método que conserva ambos invariantes: potencia y energía. Esta es la idea fundamental del esquema propuesto en las Refs. [33,34], la cual presentamos a continuación. La aproximación del campo $\Psi=\left(\psi_{j}\right) \in \mathbb{C}^{J}$ en el tiempo $t_{n}$ se almacena en la matriz $\Psi^{n} \in \mathbb{C}^{M \times J}$, para todo $m=0, \ldots, M-1$, la fila $\Psi_{(m, *)}^{n}$ representa la aproximación de $\Psi\left(x_{m}, t_{n}\right)$. Para $j=0, \ldots, J$ definimos las matrices auxiliares $\widetilde{\boldsymbol{\Psi}}^{(j, n)} \in \mathbb{C}^{M \times J}$

$$
\left\{\begin{array}{cl}
\boldsymbol{\Psi}^{n} & j=0, \\
{\left[\boldsymbol{\Psi}_{(*, 1)}^{n+1} \ldots \boldsymbol{\Psi}_{(*, j)}^{n+1} \boldsymbol{\Psi}_{(*, j+1)}^{n} \ldots \boldsymbol{\Psi}_{(*, J)}^{n}\right]} & j=1, \ldots, J-1, \\
\widetilde{\boldsymbol{\Psi}}^{n+1} & j=J,
\end{array}\right.
$$

las cuales son utilizadas exclusivamente para la descripción del método, pero no en su implementación. Además, para $j=1, \ldots, J$, sea $\mathcal{N}_{j}^{n}:=\left(\eta_{m}^{(j, n)}\right) \in \mathbb{C}^{M}$ el vector auxiliar cuya componente $m$ está definida por

$$
\eta_{m}^{(j, n)}:=\frac{F\left(\left|\widetilde{\boldsymbol{\Psi}}_{(m, *)}^{(j, n)}\right|^{2}\right)-F\left(\left|\widetilde{\boldsymbol{\Psi}}_{(m, *)}^{(j-1, n)}\right|^{2}\right)}{\left|\boldsymbol{\Psi}_{(m, j)}^{n+1}\right|^{2}-\left|\boldsymbol{\Psi}_{(m, j)}^{n}\right|^{2}} .
$$

$\underset{\text { mo }}{\text { Cuando }}\left|\Psi_{(m, j)}^{n+1}\right| \approx\left|\Psi_{(m, j)}^{n}\right|$ esta se define simplemente co-

$$
\eta_{m}^{(j, n)}:=\frac{\partial F}{\partial \xi_{j}}\left(\left|\widetilde{\boldsymbol{\Psi}}_{(m, *)}^{(j, n)}\right|^{2}\right)=f_{j}\left(\left|\boldsymbol{\Psi}_{(m, *)}^{n}\right|^{2}\right)
$$

Esta notación refleja la idea fundamental del esquema propuesto, la cual consiste en realizar una barrida de tipo GaussSeidel en las $J$ componentes del campo $\Psi$. La aproximación de la componente $j+1$ se obtiene a partir de la información recién calculada de las componentes $1, \ldots, j$. Sea $\Delta_{h}:=A^{-1} D$ la matriz asociada a una discretización de alto orden de la segunda derivada obtenida de acuerdo a la Ec. (5); utilizando los vectores, definidos para cualquier $n \in \mathbb{N}$, por

$$
\begin{aligned}
\delta_{\tau} \Psi_{j}^{n} & :=\frac{1}{\tau}\left(\Psi_{(*, j)}^{n+1}-\Psi_{(*, j)}^{n}\right), \\
\Psi_{j}^{n+1 / 2} & :=\frac{1}{2}\left(\Psi_{(*, j)}^{n+1}+\Psi_{(*, j))}^{n}\right),
\end{aligned}
$$

y el producto Hadamard $\odot$, un paso en tiempo del método propuesto, el cual será denotado por $\Phi_{\tau}(\cdot)$, se escribe en forma vectorial de la siguiente manera 
Algoritmo 1: $\Psi^{n+1}=\Phi_{\tau}\left(\Psi^{n}\right)$.

1. $\widetilde{\boldsymbol{\Psi}}^{(0, n)} \longleftarrow \boldsymbol{\Psi}^{n}$

/* Barrida Gauss-Seidel no lineal hacia adelante */

2. FOR $j=1$ TO $J$ DO

3. Calcule $\mathcal{N}_{j}^{n}$ utilizando (12)-(13)

4. Resuelva para $\Psi_{(*, j)}^{n+1} \in \mathbb{C}^{M}$ :

$$
i \delta_{\tau / 2} \Psi_{j}^{n}=-\sigma_{j} \Delta_{h} \Psi_{j}^{n+1 / 2}-\mathcal{N}_{j}^{n} \odot \Psi_{j}^{n+1 / 2},
$$

\section{END-FOR}

6. $\Psi^{n+1} \longleftarrow \widetilde{\Psi}^{(J, n)}$.

/* Barrida Gauss-Seidel no lineal hacia atrás */

7. FOR $j=J$ DOWNTO 1 DO

8. Calcule $\mathcal{N}_{j}^{n+1}$ utilizando (12)-(13)

9. Resuelva para $\Psi_{(*, j)}^{n+1} \in \mathbb{C}^{M}$ :

$$
i \delta_{\tau / 2} \Psi_{j}^{n}=-\sigma_{j} \Delta_{h} \Psi_{j}^{n+1 / 2}-\mathcal{N}_{j}^{n+1} \odot \Psi_{j}^{n+1 / 2},
$$

\section{END-FOR}

11. $\boldsymbol{\Psi}^{n+1} \longleftarrow \widetilde{\boldsymbol{\Psi}}^{(0, n+1)}$.

Avanzando con un incremento en tiempo $\tau$ en lugar de $\tau / 2$, el ciclo FOR (líneas 2-5) define un método conservativo, denotado por $\phi_{\tau}(\cdot)$, el cual posee las siguientes características:

1. El método conserva los análogos discretos de potencia y energía.

2. Para sistemas, es decir cuando $J>1$, la convergencia en tiempo es apenas lineal. Se ilustrará numéricamente que este converge de orden $p$ cuando se utiliza una discretización espacial del mismo orden.

3. Para un problema de una sola ecuación, este esquema se reduce al método DFP [9], el cual es de orden cuadrático en tiempo y en espacio, cuando se utiliza el operador de discreto $\Delta_{h}=\Delta_{0}$.

4. El segundo ciclo FOR no es otra cosa más que el operador adjunto en tiempo de $\phi_{\tau / 2}(\cdot)$, ver la Ref. [42] para la definición formal de este operador, de donde $\Phi_{\tau}(\cdot)=\phi_{\tau / 2}^{*}(\cdot) \circ \phi_{\tau / 2}(\cdot)$. Por lo tanto, se obtiene un esquema de convergencia cuadrática en tiempo.

La teoría de composición de métodos numéricos para ecuaciones diferenciales ordinarias permite desarrollar métodos de alto orden en tiempo a partir de un método base simétrico de segundo orden, manteniendo sus propiedades de conservación. Para una exposición exhaustiva sobre este tipo de métodos se sugiere consultar la Ref. [42].

En este trabajo consideramos una serie de métodos de composición simétricos de orden $2 s$ con $2 m-1$ sub-pasos, de la forma

$\Theta_{2 m-1}^{[2 s]}=\Phi_{\beta_{1} \tau} \circ \ldots \circ \Phi_{\beta_{(m-1)} \tau} \circ \Phi_{\beta_{m} \tau} \circ \Phi_{\beta_{(m-1)} \tau} \ldots \circ \Phi_{\beta_{1} \tau}$, cuyos coeficientes $\left\{\beta_{j}\right\}_{j=1}^{m} \subset \mathbb{R}$ satisfacen ciertas condiciones necesarias para alcanzar el orden deseado.

Las dos familias de métodos de composición más conocidas se deben a Yoshida [43] y a Suzuki [44]; esto debido a que sus coeficientes pueden obtenerse fácilmente de forma explícita. Para obtener métodos de orden par arbitrario, estos se definen de manera recursiva de la siguiente manera:

\section{- Yoshida:}

$$
\mathcal{Y}_{\tau}^{[2 k+2]}=\mathcal{Y}_{\beta_{1} \tau}^{[2 k]} \circ \mathcal{Y}_{\beta_{2} \tau}^{[2 k]} \circ \mathcal{Y}_{\beta_{1} \tau}^{[2 k]},
$$

donde $\mathcal{Y}_{\tau}^{[2]}:=\Phi_{\tau}$ y $\beta_{1}=\left(2-2^{1 /(2 k+1)}\right)^{-1}, \beta_{2}=$ $1-2 \beta_{1}$.

\section{- Suzuki:}

$$
\mathcal{S}_{\tau}^{[2 k+2]}=\mathcal{S}_{\beta_{1} \tau}^{[2 k]} \circ \mathcal{S}_{\beta_{2} \tau}^{[2 k]} \circ \mathcal{S}_{\beta_{3} \tau}^{[2 k]} \circ \mathcal{S}_{\beta_{2} \tau}^{[2 k]} \circ \mathcal{S}_{\beta_{1} \tau}^{[2 k]},
$$

donde $\mathcal{S}_{\tau}^{[2]}:=\Phi_{\tau}$ y $\beta_{1}=\beta_{2}=\left(4-4^{1 /(2 k+1)}\right)^{-1}$, $\beta_{3}=1-4 \beta_{1}$.

No obstante, el número de sub-pasos de los métodos de Yoshida y Suzuki se vuelve rápidamente excesivo en comparación con el número mínimo necesario para alcanzar el mismo orden; por ejemplo el método $\bullet$ de orden 6 en el Cuadro I requiere solamente 7 sub-pasos mientras que los métodos de Yoshida y Suzuki del mismo orden requieren 9 y 25 sub-pasos, respectivamente. Por otro lado dichos métodos no siempre son los más precisos entre aquellos con el mismo número de sub-pasos, por ejemplo el método $\star$ en el Cuadro I es más preciso que el método de Suzuki con el mismo orden y número de sub-pasos, véase en la Ref. [45]. Los coeficientes de algunos otros métodos de composición para órdenes específicos se encuentran descritos en el Cuadro I.

\section{Conservación de invariantes discretos}

La motivación del presente trabajo es la de desarrollar un esquema numérico que conserve los análogos discretos de la potencia y de la energía. En cada tiempo $t_{n}$, la potencia discreta de la componente $j$ y la energía global del sistema se definen, respectivamente, por

$$
\begin{aligned}
\mathcal{P}_{j, h}\left(t_{n}\right):= & \left\|\Psi^{n}(*, j)\right\|_{h}^{2}, \\
\mathcal{H}_{h}\left(t_{n}\right):= & -h \sum_{j=1}^{J} \sigma_{j}\left\langle\Delta_{h} \Psi_{(*, j)}^{n} ; \Psi_{(*, j)}^{n}\right\rangle \\
& -h \sum_{m=0}^{M-1} F\left(\left|\boldsymbol{\Psi}_{(m, *)}^{n}\right|^{2}\right),
\end{aligned}
$$




\begin{tabular}{|c|c|c|c|}
\hline Ref. & Orden & Sub-pasos & Coeficientes \\
\hline [46] & 4 & 5 & $\beta_{1}=\frac{3+\sqrt{3}}{6}, \beta_{2}=\frac{3-\sqrt{3}}{6}, \beta_{3}=-1$. \\
\hline \multirow[t]{2}{*}{$\star[45]$} & 4 & 5 & $\beta_{1}=0.28$ \\
\hline & & & $\begin{array}{l}\beta_{2}=0.62546642846767004501 \\
\beta_{3}=1-2\left(\beta_{1}+\beta_{2}\right)\end{array}$ \\
\hline \multirow[t]{2}{*}{ [47] } & 4 & 7 & $\beta_{1}=\beta_{2}=\beta_{3}=\left(6-6^{1 / 3}\right)^{-1}$ \\
\hline & & & $\beta_{4}=1-6 \beta_{1}$ \\
\hline \multirow[t]{4}{*}{ • [43] } & 6 & 7 & $\beta_{1}=0.78451361047755726382$ \\
\hline & & & $\beta_{2}=0.23557321335935813368$ \\
\hline & & & $\beta_{3}=-1.17767998417887100695$ \\
\hline & & & $\beta_{4}=1-2\left(\beta_{1}+\beta_{2}+\beta_{3}\right)$ \\
\hline \multirow[t]{5}{*}{ [45] } & 6 & 9 & $\beta_{1}=0.1867$ \\
\hline & & & $\beta_{2}=0.55549702371247839916$ \\
\hline & & & $\beta_{3}=0.12946694891347535806$ \\
\hline & & & $\beta_{4}=-0.84326562338773460855$ \\
\hline & & & $\beta_{5}=1-2\left(\beta_{1}+\beta_{2}+\beta_{3}+\beta_{4}\right)$ \\
\hline
\end{tabular}

donde, para todo $\boldsymbol{z}=\left(\zeta_{j}\right) \in \mathbb{C}^{M},\|\boldsymbol{z}\|_{h}^{2}:=h \sum_{j=0}^{M-1}\left|\zeta_{j}\right|^{2}$ es la norma $l_{2, h}$ en $\mathbb{C}^{M}$. Esta representa una versión discreta de la norma integral $\|\cdot\|$. Debemos resaltar dos aspectos importantes: el primero es que ambas cantidades discretas intentan emular las definiciones formales expresadas en las Ecs. (2a) y (2b); y el segundo, es que

$$
-h\left\langle\Delta_{h} \boldsymbol{\Psi}_{(*, j)}^{n} ; \boldsymbol{\Psi}_{(*, j)}^{n}\right\rangle \text { aproxima } \int_{a}^{b}\left|\psi_{j}\left(x, t_{n}\right)\right|^{2} \mathrm{dx},
$$

sin embargo, esta depende claramente de la fórmula de diferencias finitas utilizada.

La siguiente proposición resume las propiedades de conservación del método.

Proposición 2 (Conservación de invariantes) Para el sistema (1) con condiciones de borde periódicas, el método conserva

- la potencia discreta de cada componente del campo vectorial $\Psi$; es decir, para cualquier $j=1, \ldots, J y$ $n \in \mathbb{N}$, se tiene $\mathcal{P}_{j, h}\left(t_{n}\right)=\mathcal{P}_{j, h}(0)$.

- la energía discreta del sistema; es decir, para cualquier $n \in \mathbb{N}, \mathcal{H}_{h}\left(t_{n}\right)=\mathcal{H}_{h}(0)$.

Prueba. Puesto que se trata de un método compuesto es suficiente mostrar que el método básico, $\phi_{\tau}(\cdot)$ posee las propiedades de conservación deseadas. Sin mayor dificultad, este resultado se obtiene empleando la misma línea de razonamiento realizada en la Ref. [34]. Esta se basa, fundamentalmente en la simetría del operador de discretización de la segunda derivada. En nuestro caso, dicha propiedad fue probada en la Proposición 1 .

Proposición 3 El método propuesto es estable en la norma $l_{2, h}$.

Prueba. Esto es una consecuencia inmediata de la conservación de la potencia.

\section{Aspectos computacionales}

Para una implementación eficiente en aritmética compleja, los problemas no lineales escalares, las Ecs. (15) y (16), para cada una de las componentes del campo $\Psi$, se resuelven mediante un método semi-lineal. Esencialmente este tipo de métodos descomponen el problema original en una parte lineal, la cual se trata de manera implícita y una parte no lineal que simplemente se evalúa de manera explícita. Las principales características de este esquema son las siguientes:

1. La alta eficiencia del esquema se debe, mayormente, a no tener que ensamblar el Jacobiano del término no lineal. Este simplemente se evalúa directamente.

2. Considerando la posibilidad de una discretización implícita $\left(A \neq I_{M}\right)$, los problemas no lineales (15) y (16) se reescriben de la siguiente forma

$$
\mathcal{B} \Psi^{n+1}=\mathcal{C} \Psi^{n}-\tau A \mathcal{N}^{n} \odot\left(\Psi^{n+1}+\Psi^{n}\right) .
$$

donde

$$
\mathcal{B}:=4 i A+\tau \sigma D \quad \text { y } \quad \mathcal{C}:=4 i A-\tau \sigma D .
$$


3. Para resolver el sistema no lineal (18), asociado a cada componente del campo, se considera un esquema semi-lineal, implementado en el Algoritmo 2.
- El cálculo del vector $\mathcal{N}^{n}$ se realiza reemplazando el vector $\widetilde{\boldsymbol{\Psi}}_{(*, j)}^{n+1}$ en las expresiones (12) o (13) por $z^{(l)}$ en cada iteración $l$.
Algoritmo 2: Esquema semi lineal para resolver (18).

1. $z^{(0)} \longleftarrow \Psi^{n}$

2. Factorización $L U$ de $\mathcal{B}$

3. $b_{0} \longleftarrow \mathcal{C} \Psi^{n}$

4. FOR $l=1, \ldots$ UNTIL CONVERGENCE DO

5. $\quad b_{l} \longleftarrow A\left(\mathcal{N}^{n} \odot\left(z^{(l)}+\Psi^{n}\right)\right)$

6. $\quad b \longleftarrow b_{0}-\tau b_{l}$

7. /* Utilice la factorización $L U$ para resolver */

$$
\mathcal{B} z^{(l)}=b .
$$

8. $\quad l \longleftarrow l+1$

\section{END-FOR}

10. $\Psi^{n+1} \longleftarrow z^{(l)}$.

La existencia y unicidad de la solución del sistema lineal (20) está garantizada por el siguiente resultado

Proposición 4 La matriz $\mathcal{B}$ es no singular para cualquier incremento en tiempo $\tau>0$.

Prueba. Supóngase lo contrario, $\mathcal{B}$ singular; es decir, existe un vector no nulo, $z \in \mathbb{C}^{M}$, tal que $\mathcal{B} z=0$. De donde

$$
(4 i A+\tau \sigma D) z=0 \quad \Longleftrightarrow \quad A^{-1} D z=-\frac{4 i}{\tau \sigma} z .
$$

Por lo tanto, $-(4 i) /(\tau \sigma)$ es un valor propio de $\Delta_{h}=A^{-1} D$, lo cual es absurdo ya que por la simetría de esta matriz todos sus valores propios son reales.

Algunos comentarios adicionales

- Como resultado inmediato de considerar un incremento en tiempo $\tau$ constante, se tiene que $\mathcal{B}$ también lo es, por lo que, una factorización $L U$ o un precondicionador pueden ser utilizados. En la implementación del método, estos deben ser calculados en la fase de inicialización. Aquí se incluyó en la línea 2.

- Para problemas en 2D/3D se podría utilizar un método iterativo para la solución del problema lineal (20) y sustituir la factorización $L U$ en la línea 2 por la inicialización de un precondicionador apropiado.

- Como criterio de parada en el ciclo de la línea 4, se utilizó la norma $l_{2, h}$ de la diferencia entre dos aproximaciones consecutivas; sin embargo, otros criterios, por ejemplo, basados en la conservación de la potencia, podrían ser utilizados.

\section{Validación numérica}

Con el propósito de validar las propiedades de convergencia y conservación del método propuesto se presenta una serie de experimentos numéricos. Para ello se realizó una implementación en el ambiente MATLAB ${ }^{\circledR}$ en aritmética compleja. Puesto que la técnica propuesta es una combinación del método semilla de orden 2, formulado en el Algoritmo 1, con uno compuesto; esta se denotará por $\Phi_{\tau}$-[Ref], donde [Ref] indica la referencia donde se detalla el método compuesto.

\subsection{Ejemplo 1}

Como primer ejemplo se considera un sistema de dos componentes, definido en $\Omega=(-40,40)$ por

$$
\left\{\begin{array}{l}
i \psi_{1, t}=-\frac{1}{2} \psi_{1, x x}-\left(\left|\psi_{1}\right|^{2}+\frac{2}{3}\left|\psi_{2}\right|^{2}\right) \psi_{1} \\
i \psi_{2, t}=-\frac{1}{2} \psi_{2, x x}-\left(\frac{2}{3}\left|\psi_{1}\right|^{2}+\left|\psi_{2}\right|^{2}\right) \psi_{2}
\end{array}\right.
$$

y condiciones iniciales

$$
\begin{aligned}
& \psi_{1}^{o}(x)=\sqrt{2} \operatorname{sech}(x+10) e^{i x}, \\
& \psi_{2}^{o}(x)=\sqrt{2} \operatorname{sech}(x-10) e^{-i x} .
\end{aligned}
$$

Primero validamos la convergencia del método propuesto. En el Cuadro II se muestran los órdenes de convergencia estimados (OCE) para distintos métodos de composición de cuarto orden en combinación con discretizaciones espaciales del mismo orden, utilizando las fórmulas de diferencias finitas (8a) y (8b). Las tasas de convergencia fueron calculadas con respecto a una solución de referencia obtenida con el método de Suzuki de cuarto orden [44] con $h_{r e f}=1 / 160 \mathrm{y}$ $\tau_{r e f}=h_{r e f} / 2$. El error para el tiempo final $T=1$ se calculó de la siguiente forma

$$
\text { Error }:=\sqrt{\sum_{j=1}^{J}\left\|\Psi_{j}-\Psi_{j, r e f}\right\|_{h}^{2}} .
$$

En el Cuadro III, se muestran las tasas de convergencia para el método propuesto, $\Phi_{\tau^{-}}$[43], de sexto orden y 7 subpasos, combinado con discretizaciones espaciales del mismo 
TABLA II. Precisión de los métodos de cuarto orden para el Ejemplo 1.

\begin{tabular}{|c|c|c|c|c|c|c|c|c|}
\hline \multirow[b]{2}{*}{$h$} & \multicolumn{2}{|c|}{$\Phi_{\tau^{-}}[43]$} & \multicolumn{2}{|c|}{$\Phi_{\tau^{-}}[44]$} & \multicolumn{2}{|c|}{$\Phi_{\tau^{-}}[45]$} & \multicolumn{2}{|c|}{$\Phi_{\tau^{-}}[46]$} \\
\hline & Error & OCE & Error & OCE & Error & OCE & Error & $\mathrm{OCE}$ \\
\hline \multicolumn{9}{|c|}{ Fórmula de diferencias finitas (8a) } \\
\hline $2.00 \times 10^{-1}$ & $4.7039 \mathrm{e}-03$ & - & $4.5626 \mathrm{e}-03$ & - & $4.5645 \mathrm{e}-03$ & - & $4.5804 \mathrm{e}-03$ & - \\
\hline $1.00 \times 10^{-1}$ & $3.6103 e-04$ & 3.70 & $2.9754 \mathrm{e}-04$ & 3.94 & $2.9820 \mathrm{e}-04$ & 3.93 & $3.0038 \mathrm{e}-04$ & 3.93 \\
\hline $5.00 \times 10^{-2}$ & $2.7561 \mathrm{e}-05$ & 3.71 & $1.8837 \mathrm{e}-05$ & 3.98 & $1.8891 \mathrm{e}-05$ & 3.98 & $1.9072 \mathrm{e}-05$ & 3.98 \\
\hline $2.50 \times 10^{-2}$ & $1.9067 \mathrm{e}-06$ & 3.85 & $1.1771 \mathrm{e}-06$ & 4.00 & $1.1807 \mathrm{e}-06$ & 4.00 & $1.1930 \mathrm{e}-06$ & 3.99 \\
\hline $1.25 \times 10^{-2}$ & $1.2031 \mathrm{e}-07$ & 3.99 & $6.9306 \mathrm{e}-08$ & 4.08 & $6.9536 \mathrm{e}-08$ & 4.08 & $7.0329 \mathrm{e}-08$ & 4.08 \\
\hline \multicolumn{9}{|c|}{ Fórmula de diferencias finitas $(8 \mathrm{~b})$} \\
\hline $2.00 \times 10^{-1}$ & $2.2277 \mathrm{e}-03$ & - & $1.8875 \mathrm{e}-03$ & - & $1.8918 \mathrm{e}-03$ & - & $1.9161 \mathrm{e}-03$ & - \\
\hline $1.00 \times 10^{-1}$ & $2.1848 \mathrm{e}-04$ & 3.35 & $1.1502 \mathrm{e}-04$ & 4.04 & $1.1587 \mathrm{e}-04$ & 4.02 & $1.1896 \mathrm{e}-04$ & 4.00 \\
\hline $5.00 \times 10^{-2}$ & $1.9956 \mathrm{e}-05$ & 3.45 & 7.1411e-06 & 4.01 & $7.2091 \mathrm{e}-06$ & 4.01 & $7.4720 \mathrm{e}-06$ & 3.99 \\
\hline $2.50 \times 10^{-2}$ & $1.4748 \mathrm{e}-06$ & 3.76 & $4.4135 \mathrm{e}-07$ & 4.02 & $4.4589 \mathrm{e}-07$ & 4.01 & $4.6419 \mathrm{e}-07$ & 4.00 \\
\hline $1.25 \times 10^{-2}$ & $9.5453 \mathrm{e}-08$ & 3.95 & $2.3248 \mathrm{e}-08$ & 4.24 & $2.3549 \mathrm{e}-08$ & 4.24 & $2.4826 \mathrm{e}-08$ & 4.22 \\
\hline
\end{tabular}

TABLA III. Precisión de los métodos de sexto orden para el Ejemplo 1.

\begin{tabular}{ccccc}
\hline & \multicolumn{2}{c}{$\Phi_{\tau}-[43] / \Delta_{h}$-Explícita } & \multicolumn{2}{c}{$\Phi_{\tau}$ - $[43] / \Delta_{h}$-Compacta } \\
Error & OCE & - & Error & - \\
\hline $4.00 \times 10^{-1}$ & $4.0033 \mathrm{e}-02$ & $\mathbf{5 . 6 4}$ & $1.7123 \mathrm{e}-02$ & $1.8656 \mathrm{e}-04$ \\
\\
$2.00 \times 10^{-1}$ & $8.0437 \mathrm{e}-04$ & $\mathbf{5 . 7 6}$ & $2.7535 \mathrm{e}-06$ & $\mathbf{6 . 5 2}$ \\
$1.00 \times 10^{-1}$ & $1.4850 \mathrm{e}-05$ & $\mathbf{5 . 9 4}$ & $4.1615 \mathrm{e}-08$ & $\mathbf{6 . 0 8}$ \\
$5.00 \times 10^{-2}$ & $2.4213 \mathrm{e}-07$ & $\mathbf{5 . 9 9}$ & $6.3770 \mathrm{e}-10$ & $\mathbf{6 . 0 5}$ \\
$2.50 \times 10^{-2}$ & $3.8226 \mathrm{e}-09$ & $\mathbf{6 . 0 2}$ & \\
\hline
\end{tabular}

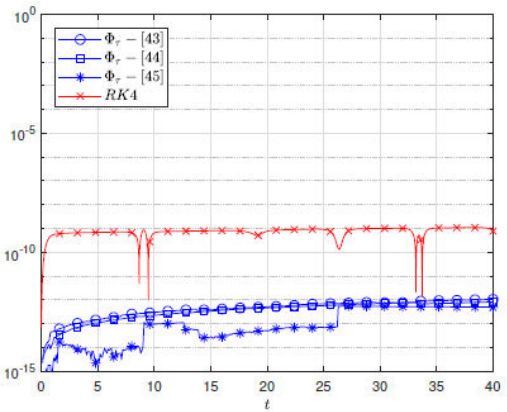

a) $\left|\mathcal{P}_{1, h}(0)-\mathcal{P}_{1, h}(t)\right|$

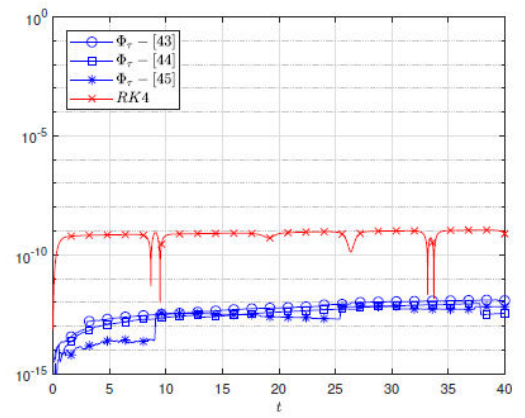

b) $\left|\mathcal{P}_{2, h}(0)-\mathcal{P}_{2, h}(t)\right|$

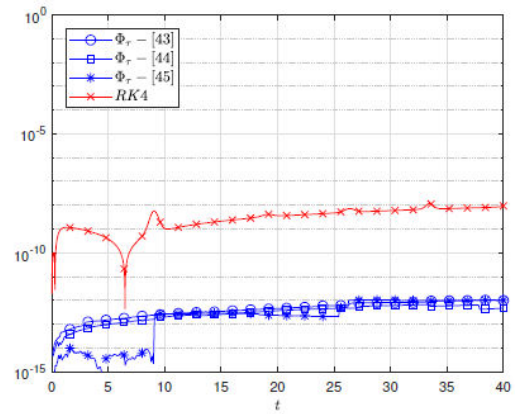

c) $\left|\mathcal{H}_{h}(0)-\mathcal{H}_{h}(t)\right|$

FIGURA 1. Evolución del error de los invariantes discretos del Ejemplo 1.

orden, dadas por la fórmula compacta (9a) y la fórmula explícita

$$
\delta_{h}^{2}\left(\psi_{i}\right):=\frac{1}{180 h^{2}} \sum_{j=0}^{3} \beta_{j} \psi\left(x_{i \pm j}\right)
$$

donde

$$
\beta_{0}=-490, \beta_{1}=270, \beta_{2}=-27, \beta_{3}=2,
$$

para la cual $\mathcal{B}$ es una matriz heptadiagonal. Estas tasas fueron calculadas con una solución de referencia con paramétros de discretización $h_{r e f}=1 / 320$ y $\tau_{r e f}=h_{r e f} / 4$. Puesto que en ambos cuadros, se consideró para cada una de las mallas, un incremento en tiempo $\tau$ proporcional a $h$, se validan los órdenes de convergencia esperados, tanto en tiempo como en espacio.

Para comprobar las propiedades conservativas del método propuesto, estudiamos la evolución del error en los invariantes discretos, con respecto a su valor inicial. Con el único propósito de comparación, hemos incluido, en la Fig. 1, la evolución del error de estos invariantes utilizando el método 


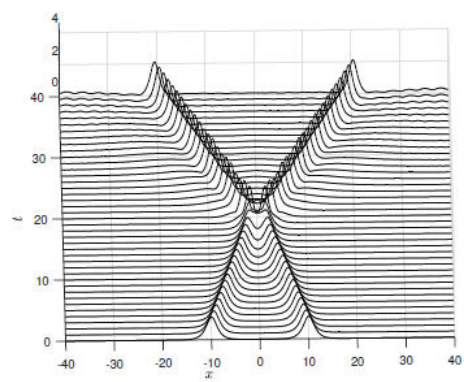

a) $\alpha=1 / 2$

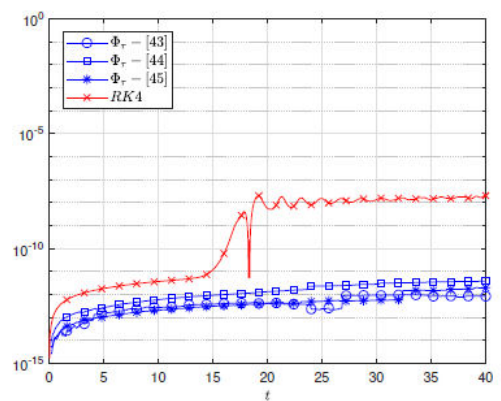

d) $\left|\mathcal{P}_{1, h}(0)-\mathcal{P}_{1, h}(t)\right|, \alpha=2$

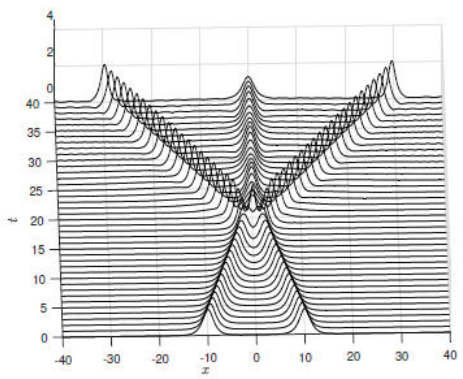

b) $\alpha=1$

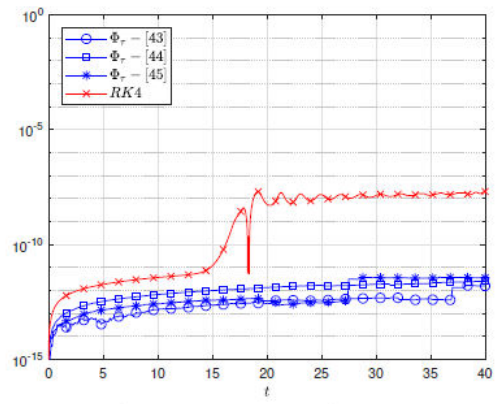

e) $\left|\mathcal{P}_{2, h}(0)-\mathcal{P}_{2, h}(t)\right|, \alpha=2$

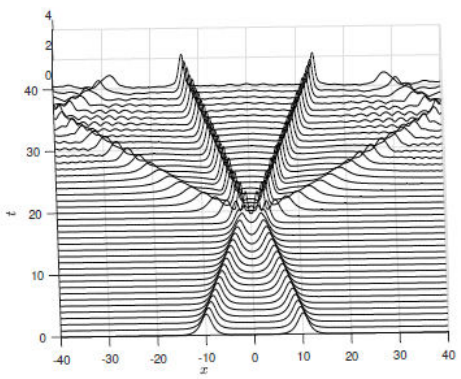

c) $\alpha=2$

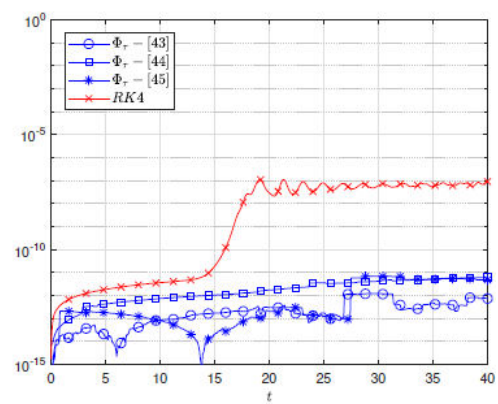

f) $\left|\mathcal{H}_{h}(0)-\mathcal{H}_{h}(t)\right|, \alpha=2$

FIGURA 2. Soluciones y evolución del error de los invariantes discretos del Ejemplo 2.

explícito de Runge-Kutta de cuarto orden (RK4), el cual no es conservativo. Nótese un aumento de hasta cuatro órdenes de magnitud en el error, con respecto a los resultados obtenidos con el método propuesto combinado con diversos métodos de composición de cuarto orden. En los experimentos numéricos se usó la fórmula compacta (8b) para discretizar el operador de segundo orden, en una malla uniforme con $h=0.2$. Para los métodos conservativos el incremento en tiempo se tomó $\tau=h / 2$; mientras que para el método RK4 se tuvo que usar un incremento en tiempo de orden $h^{2}(\tau=0.005)$ para poder satisfacer las condiciones de estabilidad requeridas por el método explícito RK4, las cuales fueron analizadas por Caplan y Carrero-González en [48] para una ecuación de Schödinger no lineal con potencial cúbico.

\subsection{Ejemplo 2}

Como segundo ejemplo se considera un sistema de dos componentes, que fue estudiado en $[16,17,24]$. Este sistema depende de un parámetro real $\alpha$ el cual influye en el número de ondas que se forman después de una colisión. El sistema está definido en $\Omega=(-40,40)$ por

$$
\left\{\begin{array}{l}
i \psi_{1, t}=-\psi_{1, x x}-\left(\left|\psi_{1}\right|^{2}+(1+2 \alpha)\left|\psi_{2}\right|^{2}\right) \psi_{1}, \\
i \psi_{2, t}=-\psi_{2, x x}-\left((1+2 \alpha)\left|\psi_{1}\right|^{2}+\left|\psi_{2}\right|^{2}\right) \psi_{2},
\end{array}\right.
$$

y condiciones iniciales

$$
\begin{aligned}
& \psi_{1}^{o}(x)=\sqrt{2} \operatorname{sech}(x+10) e^{i x / 4}, \\
& \psi_{2}^{o}(x)=\sqrt{2} \operatorname{sech}(x-10) e^{-i x / 4} .
\end{aligned}
$$

En las Figs. 2a)-2c) se ilustra el comportamiento de $\left|\Psi_{1}\right|+$ $\left|\Psi_{2}\right|$, para los siguientes valores de $\alpha$ : a) $\alpha=1 / 2$, b) $\alpha=1$ and c) $\alpha=2$, donde se aprecia la generación de 2, 3 y 4 ondas, respectivamente, con el crecimiento del parámetro $\alpha$. Las propiedades conservativas del método propuesto para este ejemplo con $\alpha=2$ se ilustran en las Figs. 2d) y 2e) para la potencia de cada componente y en la Fig. 2f) para la energía del sistema. Resultados similares fueron obtenidos para otros valores de $\alpha$. Los cálculos fueron obtenidos con $h=0.2$ y $\tau=h / 2=0.1$ para los métodos de composición y con $\tau=0.005$ para el método RK4. A diferencia de otros esquemas en tiempo como el reportado en [16] y el método RK4, en nuestro método, la conservación de los invariantes no se ve influenciada por la creación de ondas, la cual sucede aproximadamente en el tiempo $t=20$. Por otro lado, nótese una vez más la restricción en tiempo del método RK4 y la poca precisión en la conservación.

En los Cuadros IV y V se muestran los órdenes de convergencia para diferentes métodos de composición de cuarto y sexto orden, respectivamente, en combinación con discretizaciones espaciales del mismo orden. Las tasas de convergencia fueron calculadas para el sistema de Manakov, $\alpha=0 \mathrm{y}$ coeficiente de dispersión $\sigma=1 / 2$, para la solución exacta dada por

$$
\begin{aligned}
& \psi_{1}(x, t)=\operatorname{sech}(\sqrt{2}(x-t)) e^{i(x+t / 2)} \\
& \psi_{2}(x, t)=\operatorname{sech}(\sqrt{2}(x-t)) e^{i(x+t / 2)} .
\end{aligned}
$$

\subsection{Ejemplo 3}

El objetivo de este experimento es estudiar el efecto del parámetro $\gamma$ en la conservación de los invariantes. Para ello, 


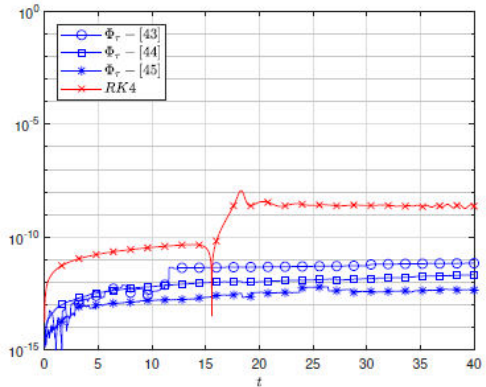

a) $\left|\mathcal{P}_{1, h}(t)-\mathcal{P}_{1, h}(0)\right|$ para $\gamma=1$

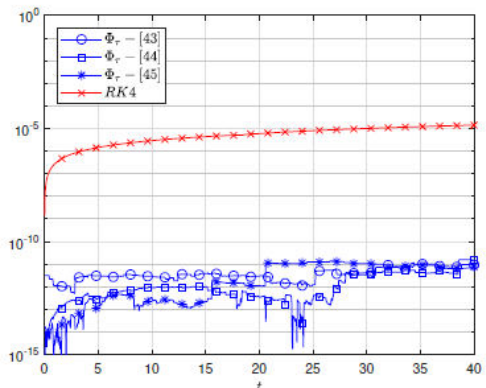

d) $\left|\mathcal{P}_{1, h}(t)-\mathcal{P}_{1, h}(0)\right|$ para $\gamma=10$

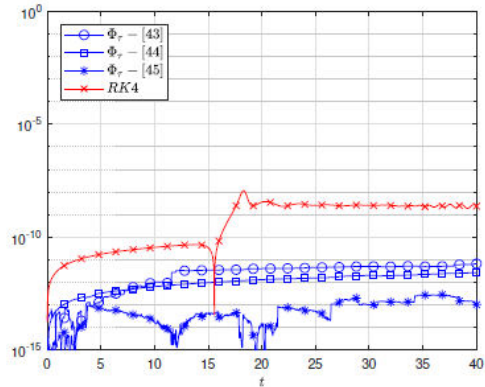

b) $\left|\mathcal{P}_{2, h}(t)-\mathcal{P}_{2, h}(0)\right|$ para $\gamma=1$

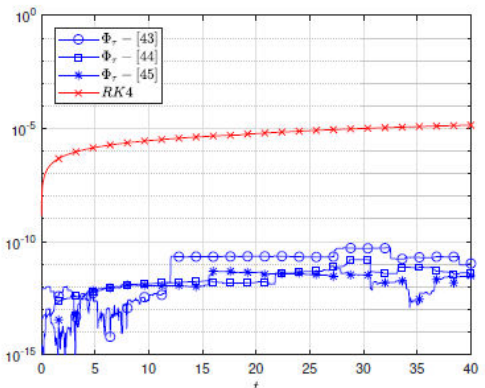

e) $\left|\mathcal{P}_{2, h}(t)-\mathcal{P}_{2, h}(0)\right| \operatorname{para} \gamma=10$

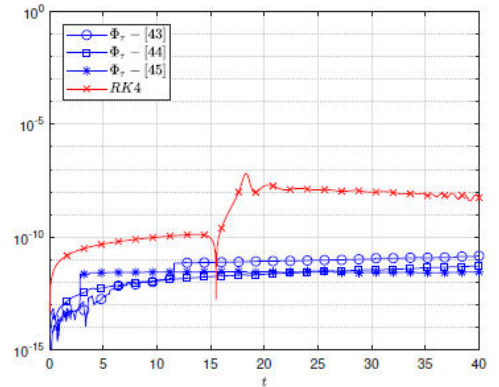

c) $\left|\mathcal{H}_{h}(t)-\mathcal{H}_{h}(0)\right|$ para $\gamma=1$

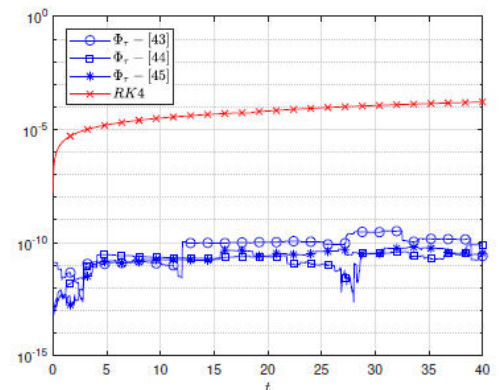

f) $\left|\mathcal{H}_{h}(t)-\mathcal{H}_{h}(0)\right| \operatorname{para} \gamma=10$

FIGURE 3. Evolución del error de los invariantes discretos del Ejemplo 3.

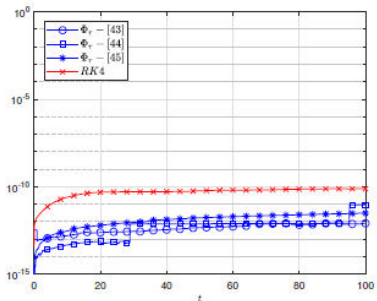

a) $\left|\mathcal{P}_{1, h}(t)-\mathcal{P}_{1, h}(0)\right|$

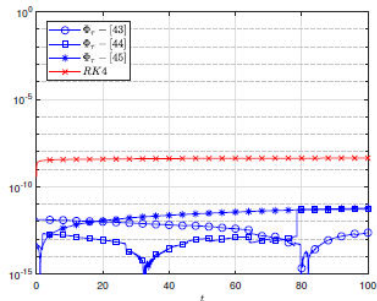

b) $\left|\mathcal{P}_{2, h}(t)-\mathcal{P}_{2, h}(0)\right|$

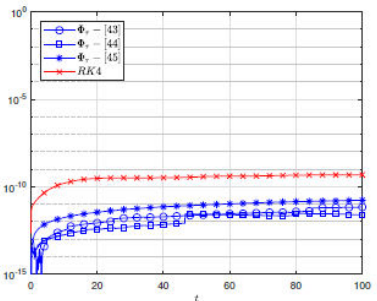

c) $\left|\mathcal{P}_{3, h}(t)-\mathcal{P}_{3, h}(0)\right|$

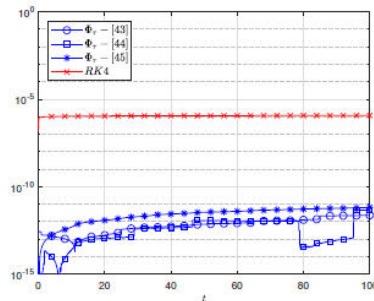

d) $\left|\mathcal{H}_{h}(t)-\mathcal{H}_{h}(0)\right|$

FIGURE 4. Evolución del error de los invariantes discretos del Ejemplo 4.

TABLA IV. Precisión de los métodos de cuarto orden para el sistema de Manakov.

\begin{tabular}{|c|c|c|c|c|c|c|c|c|}
\hline \multirow[b]{2}{*}{$h$} & \multicolumn{2}{|c|}{$\Phi_{\tau}-[43]$} & \multicolumn{2}{|c|}{$\Phi_{\tau-[44]}$} & \multicolumn{2}{|c|}{$\Phi_{\tau-}-[45]$} & \multicolumn{2}{|c|}{$\Phi_{\tau-[46]}$} \\
\hline & Error & orden & Error & orden & Error & orden & Error & orden \\
\hline \multicolumn{9}{|c|}{ Fórmula de diferencias finitas (8a) } \\
\hline $2.00 \times 10^{-1}$ & $3.9797 \mathrm{e}-03$ & - & $4.0140 \mathrm{e}-03$ & - & $4.0137 \mathrm{e}-03$ & - & $4.0122 \mathrm{e}-03$ & - \\
\hline $1.00 \times 10^{-1}$ & $2.5851 \mathrm{e}-04$ & 3.94 & $2.5951 \mathrm{e}-04$ & 3.95 & $2.5950 \mathrm{e}-04$ & 3.95 & $2.5944 \mathrm{e}-04$ & 3.95 \\
\hline $5.00 \times 10^{-2}$ & $1.6389 \mathrm{e}-05$ & 3.98 & $1.6397 \mathrm{e}-05$ & 3.98 & $1.6396 \mathrm{e}-05$ & 3.98 & $1.6395 \mathrm{e}-05$ & 3.98 \\
\hline $2.50 \times 10^{-2}$ & $1.0287 \mathrm{e}-06$ & 4.00 & $1.0278 \mathrm{e}-06$ & 4.00 & $1.0277 \mathrm{e}-06$ & 4.00 & $1.0277 \mathrm{e}-06$ & 4.00 \\
\hline $1.25 \times 10^{-2}$ & $6.4368 \mathrm{e}-08$ & 4.00 & $6.4280 \mathrm{e}-08$ & 4.00 & $6.4281 \mathrm{e}-08$ & 4.00 & $6.4278 \mathrm{e}-08$ & 4.00 \\
\hline \multicolumn{9}{|c|}{ Fórmula de diferencias finitas $(8 \mathrm{~b})$} \\
\hline $2.00 \times 10^{-1}$ & $1.6029 \mathrm{e}-03$ & - & $1.6309 \mathrm{e}-03$ & - & $1.6305 \mathrm{e}-03$ & - & $1.6291 \mathrm{e}-03$ & - \\
\hline $1.00 \times 10^{-1}$ & $9.9284 \mathrm{e}-05$ & 4.01 & $9.9464 \mathrm{e}-05$ & 4.04 & $9.9456 \mathrm{e}-05$ & 4.04 & $9.9407 \mathrm{e}-05$ & 4.03 \\
\hline $5.00 \times 10^{-2}$ & $6.2490 \mathrm{e}-06$ & 3.99 & $6.1828 \mathrm{e}-06$ & 4.01 & $6.1826 \mathrm{e}-06$ & 4.00 & $6.1813 e-06$ & 4.00 \\
\hline $2.50 \times 10^{-2}$ & $3.9213 \mathrm{e}-07$ & 3.99 & $3.8592 \mathrm{e}-07$ & 4.00 & $3.8591 \mathrm{e}-07$ & 4.00 & $3.8586 \mathrm{e}-07$ & 4.00 \\
\hline $1.25 \times 10^{-2}$ & $2.4540 \mathrm{e}-08$ & 3.99 & $2.4112 \mathrm{e}-08$ & 4.00 & $2.4111 \mathrm{e}-08$ & 4.00 & $2.4109 \mathrm{e}-08$ & 4.00 \\
\hline
\end{tabular}


TABla V. Precisión de los métodos de sexto orden para el sistema de Manakov.

\begin{tabular}{|c|c|c|c|c|}
\hline \multirow[b]{2}{*}{$h$} & \multicolumn{2}{|c|}{$\Phi_{\tau^{-}}[43]$} & \multicolumn{2}{|c|}{$\Phi_{\tau^{-}}[45]$} \\
\hline & Error & orden & Error & orden \\
\hline \multicolumn{5}{|c|}{ Fórmula de diferencias finitas (22) } \\
\hline $4.00 \times 10^{-1}$ & $2.1627 \mathrm{e}-02$ & - & $2.1585 \mathrm{e}-02$ & - \\
\hline $2.00 \times 10^{-1}$ & $4.2445 \mathrm{e}-04$ & 5.67 & $4.2389 \mathrm{e}-04$ & 5.67 \\
\hline $1.00 \times 10^{-1}$ & $7.4383 \mathrm{e}-06$ & 5.83 & $7.4177 \mathrm{e}-06$ & 5.83 \\
\hline $5.00 \times 10^{-2}$ & $1.2008 \mathrm{e}-07$ & 5.95 & $1.1953 \mathrm{e}-07$ & 5.96 \\
\hline $2.50 \times 10^{-2}$ & $1.8946 \mathrm{e}-09$ & 5.99 & $1.8823 \mathrm{e}-09$ & 5.99 \\
\hline $1.25 \times 10^{-2}$ & $3.0259 \mathrm{e}-11$ & 5.97 & $3.1360 \mathrm{e}-11$ & 5.91 \\
\hline \multicolumn{5}{|c|}{ Fórmula de diferencias finitas (9a) } \\
\hline $4.00 \times 10^{-1}$ & $8.0399 \mathrm{e}-03$ & - & $7.9885 \mathrm{e}-03$ & - \\
\hline $2.00 \times 10^{-1}$ & $9.0703 \mathrm{e}-05$ & 6.47 & $8.9873 \mathrm{e}-05$ & 6.47 \\
\hline $1.00 \times 10^{-1}$ & $1.3683 \mathrm{e}-06$ & 6.05 & $1.3414 \mathrm{e}-06$ & 6.07 \\
\hline $5.00 \times 10^{-2}$ & $2.1568 \mathrm{e}-08$ & 5.99 & $2.0727 \mathrm{e}-08$ & 6.02 \\
\hline $2.50 \times 10^{-2}$ & $3.4111 \mathrm{e}-10$ & 5.98 & $3.2333 \mathrm{e}-10$ & 6.00 \\
\hline $1.25 \times 10^{-2}$ & $5.8139 \mathrm{e}-12$ & 5.88 & $6.3729 \mathrm{e}-12$ & 5.66 \\
\hline
\end{tabular}

consideramos el siguiente sistema de dos componentes, definido en $\Omega=(-40,40) \times(0,40]$,

$$
\left\{\begin{aligned}
i \psi_{1, t} & =-\psi_{1, x x}-\left(\gamma+\left|\psi_{1}\right|^{2}+3\left|\psi_{2}\right|^{2}\right) \psi_{1} \\
i \psi_{2, t} & =-\psi_{2, x x}-\left(\gamma+3\left|\psi_{1}\right|^{2}+\left|\psi_{2}\right|^{2}\right) \psi_{2}
\end{aligned}\right.
$$

Las condiciones iniciales están dadas por,

$$
\begin{aligned}
& \psi_{1}^{o}(x)=\sqrt{2} \operatorname{sech}(x+10) e^{i x / 4}, \\
& \psi_{2}^{o}(x)=\sqrt{2} \operatorname{sech}(x-10) e^{-i x / 4} .
\end{aligned}
$$

Los parámetros de discretización utilizados fueron $h=0.2$ y $\tau=h / 4=0.05$ para los métodos de composición; por condiciones de estabilidad, $\tau=0.005$ para el método RK4. La evolución del error en los invariantes se muestra en las Figs. 3a)-3c) para $\gamma=1$ y en las Fig. 3d)-3f) para $\gamma=10$. Las escalas de precisión ratifican que el avance en tiempo propuesto en combinación con distintos métodos de composición de orden 4 es conservativo, a diferencia del método RK4. Por otro lado, es importante resaltar que, a diferencia de otros métodos como el presentado en Ref. [16], el cual pierde conservación de la energía para valores grandes de $\gamma$, nuestro método no parece ser afectado por las variaciones de dicho parámetro. Nótese además que para el método RK4, la pérdida de precisión en la conservación de todos los invariantes es bastante considerable.

\subsection{Ejemplo 4}

En este ejemplo se validan las propiedades de conservación, en un sistema de 3 componentes, definido en $(-4 \pi, 4 \pi) \times$
$(0,100]$ por

$\left\{\begin{array}{l}i \psi_{1, t}=-\psi_{1, x x}-\left(\left|\psi_{1}\right|^{2}+\frac{2}{3}\left|\psi_{2}\right|^{2}+\left|\psi_{3}\right|^{2}\right) \psi_{1} \\ i \psi_{2, t}=-\psi_{2, x x}-\left(\frac{2}{3}\left|\psi_{1}\right|^{2}+\left|\psi_{2}\right|^{2}+\frac{2}{3}\left|\psi_{3}\right|^{2}\right) \psi_{2} \\ i \psi_{3, t}=-\psi_{3, x x}-\left(\left|\psi_{1}\right|^{2}+\frac{2}{3}\left|\psi_{2}\right|^{2}+\left|\psi_{3}\right|^{2}\right) \psi_{3}\end{array}\right.$

y condiciones iniciales

$$
\left\{\begin{array}{l}
\psi_{1}(x, 0)=0.2(1-0.4 \cos (0.5 x)) \\
\psi_{2}(x, 0)=0.3(1-0.4 \cos [0.5(x+7 \pi / 4)]) \\
\psi_{3}(x, 0)=0.5(1-0.4 \cos (0.5 x))
\end{array}\right.
$$

En la Fig. 4, se ilustra la evolución del error de los invariantes discretos. Nótese que a pesar de no ser conservativo, el método RK4 preserva la potencia de cada una de las componentes del sistema bastante bien; sin embargo, no logra conservar la energía con la misma precisión del método propuesto. Estos cálculos se obtuvieron con $h=8 \pi / 320, \tau=0.04 \approx h / 2$ para los métodos $\Phi_{\tau}$-[Ref] y $\tau=0.002$ para RK4.

\subsection{Ejemplo 5}

El objetivo principal de este ejemplo es el verificar las propiedades del método propuesto en un sistema con potencial no lineal de saturación. Para ello se considera un sistema de 2 componentes [49] definido en $(-40,40)$ por

$$
\left\{\begin{array}{l}
i \psi_{1, t}=-\frac{1}{2} \psi_{1, x x}-\frac{\left|\psi_{1}\right|^{2}+\left|\psi_{2}\right|^{2}}{1+s\left(\left|\psi_{1}\right|^{2}+\left|\psi_{2}\right|^{2}\right)} \psi_{1} \\
i \psi_{2, t}=-\frac{1}{2} \psi_{2, x x}-\frac{\left|\psi_{1}\right|^{2}+\left|\psi_{2}\right|^{2}}{1+s\left(\left|\psi_{1}\right|^{2}+\left|\psi_{2}\right|^{2}\right)} \psi_{2}
\end{array}\right.
$$




\begin{tabular}{|c|c|c|c|c|c|c|c|c|}
\hline \multirow[b]{2}{*}{$h$} & \multicolumn{2}{|c|}{$\Phi_{\tau^{-}}[43]$} & \multicolumn{2}{|c|}{$\Phi_{\tau^{-}}[44]$} & \multicolumn{2}{|c|}{$\Phi_{\tau^{-}}[45]$} & \multicolumn{2}{|c|}{$\Phi_{\tau^{-}}[46]$} \\
\hline & Error & OCE & Error & OCE & Error & OCE & Error & OCE \\
\hline \multicolumn{9}{|c|}{ Fórmula de diferencias finitas (8a) } \\
\hline $8.00 \times 10^{-1}$ & $6.4682 \mathrm{e}-02$ & - & $6.4658 \mathrm{e}-02$ & - & $6.4658 \mathrm{e}-02$ & - & $6.4659 \mathrm{e}-02$ & - \\
\hline $4.00 \times 10^{-1}$ & $8.3155 \mathrm{e}-03$ & 2.96 & $8.2376 \mathrm{e}-03$ & 2.97 & $8.2390 \mathrm{e}-03$ & 2.97 & $8.2427 \mathrm{e}-03$ & 2.97 \\
\hline $2.00 \times 10^{-1}$ & $6.7292 \mathrm{e}-04$ & 3.63 & $6.5380 \mathrm{e}-04$ & 3.66 & $6.5411 \mathrm{e}-04$ & 3.65 & $6.5502 \mathrm{e}-04$ & 3.65 \\
\hline $1.00 \times 10^{-1}$ & $4.4665 \mathrm{e}-05$ & 3.91 & $4.3082 \mathrm{e}-05$ & 3.92 & $4.3106 \mathrm{e}-05$ & 3.92 & $4.3177 \mathrm{e}-05$ & 3.92 \\
\hline $5.00 \times 10^{-2}$ & $2.8346 \mathrm{e}-06$ & 3.98 & $2.7309 \mathrm{e}-06$ & 3.98 & $2.7330 \mathrm{e}-06$ & 3.98 & $2.7366 \mathrm{e}-06$ & 3.98 \\
\hline \multicolumn{9}{|c|}{ Fórmula de diferencias finitas (8b) } \\
\hline $8.00 \times 10^{-1}$ & $4.2762 \mathrm{e}-02$ & - & $4.2706 \mathrm{e}-02$ & - & $4.2707 \mathrm{e}-02$ & - & $4.2709 \mathrm{e}-02$ & - \\
\hline $4.00 \times 10^{-1}$ & $4.3859 \mathrm{e}-03$ & 3.29 & $4.2606 \mathrm{e}-03$ & 3.33 & $4.2628 \mathrm{e}-03$ & 3.33 & $4.2691 \mathrm{e}-03$ & 3.32 \\
\hline $2.00 \times 10^{-1}$ & $2.9251 \mathrm{e}-04$ & 3.91 & $2.7018 \mathrm{e}-04$ & 3.98 & $2.7054 \mathrm{e}-04$ & 3.98 & $2.7159 \mathrm{e}-04$ & 3.97 \\
\hline $1.00 \times 10^{-1}$ & $1.8263 \mathrm{e}-05$ & 4.00 & $1.6572 \mathrm{e}-05$ & 4.03 & $1.6598 \mathrm{e}-05$ & 4.03 & $1.6670 \mathrm{e}-05$ & 4.03 \\
\hline $5.00 \times 10^{-2}$ & $1.1457 \mathrm{e}-06$ & 4.00 & $1.0372 \mathrm{e}-06$ & 4.00 & $1.0398 \mathrm{e}-06$ & 4.00 & $1.0435 \mathrm{e}-06$ & 4.00 \\
\hline
\end{tabular}

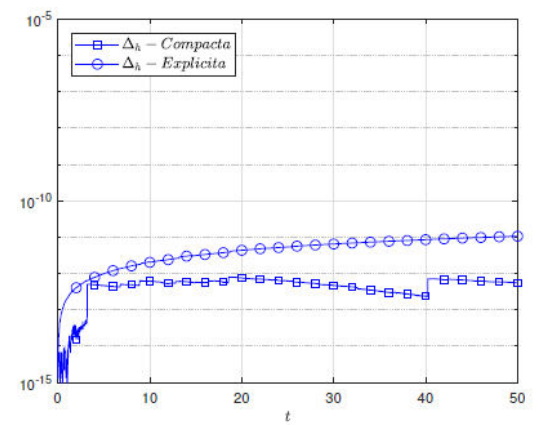

a) $\left|\mathcal{P}_{1, h}(0)-\mathcal{P}_{1, h}(t)\right|$

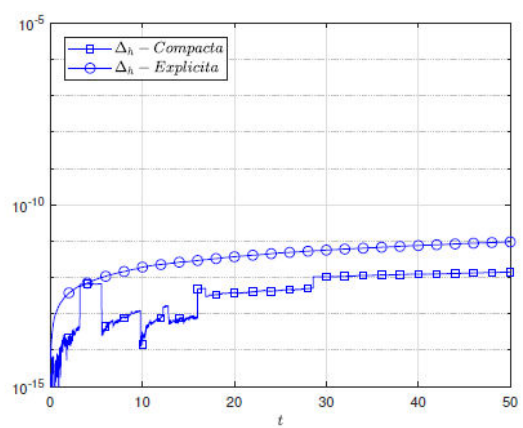

b) $\left|\mathcal{P}_{2, h}(0)-\mathcal{P}_{2, h}(t)\right|$

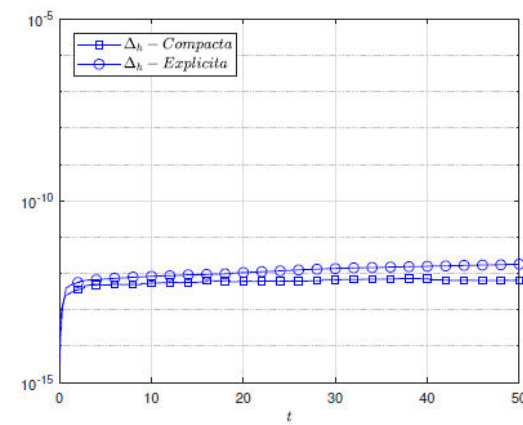

c) $\left|\mathcal{H}_{h}(0)-\mathcal{H}_{h}(t)\right|$

FIGURE 5. Evolución del error de los invariantes discretos del Ejemplo 5.

y condiciones iniciales

$$
\begin{aligned}
& \psi_{1}^{o}(x)=\sqrt{2} \operatorname{sech}(x+10) e^{i x / 4}, \\
& \psi_{2}^{o}(x)=\sqrt{2} \operatorname{sech}(x-10) e^{-i x / 4} .
\end{aligned}
$$

Para este problema el potencial $F\left(\xi_{1}, \xi_{2}\right)$ esta dado por

$$
F\left(\xi_{1}, \xi_{2}\right):=\frac{1}{s}\left(\xi_{1}+\xi_{2}\right)-\frac{1}{s^{2}} \ln \left|1+s\left(\xi_{1}+\xi_{2}\right)\right| .
$$

Los órdenes de convergencia estimados en espacio se validaron para distintos métodos de composición de cuarto orden en combinación con discretizaciones espaciales de igual orden. Las tasas de convergencia fueron calculadas con una solución de referencia obtenida con el método compuesto de sexto orden presentado en [43], con $h_{\text {ref }}=1 / 320$ y $\tau_{\text {ref }}=$ $h_{\text {ref }} / 4$. Puesto que para cada una de las mallas se consideró un incremento en tiempo proporcional a $h, \tau=h / 4$, las tasas calculadas muestran que el método es de cuarto orden en tiempo y en espacio.

La conservación de invariantes se ilustra en la Fig. 5, para el método de sexto orden $\Phi_{\tau^{-}}$[43] con la discretización es- pacial compacta (9a) y la explícita (22); y parámetros de discretización $h=0.1$ y $\tau=h / 4=0.025$. Resultados similares se obtuvieron con otros métodos.

\section{Conclusiones}

Hemos presentado un método de alto orden en tiempo y en espacio para una clase de sistemas de Schrödinger no lineales, el cual tiene la propiedad de conservar, simultáneamente, los invariantes discretos de potencia y energía. Se realiza una descomposición del sistema global inicial, en una secuencia de problemas no lineales de menor tamaño asociados a cada una de las componentes del campo. Con el único propósito de mejorar la eficiencia del método se presentó una técnica semi-lineal para resolver dichos problemas auxiliares. La extensión del método propuesto a otro tipo de problemas, como por ejemplo aquellos en donde se incluye un parámetro de desafinación en la velocidad de grupo será considerado en trabajos futuros, así como la comparación con otros métodos diseñados para sistemas. 
1. S. López, M. Esparza, G. Lem-Carrillo, y J. Gutiérrez, “Ondas solitarias no lineales: una introducción a los solitones ópticos espaciales," Rev. Mex. Fis. E 60 (2014) 39.

2. W. Liu y E. Kengne, Schrödinger equations in nonlinear systems. (Springer, Singapore, 2019), https:// doi .org/10. 1007/978-981-13-6581-2

3. T. A. Sulaiman, T. Aktürk, H. Bulut y H. M. Baskonus, Investigation of various soliton solutions to the Heisenberg ferromagnetic spin chain equation, J. Electromagn. Waves Appl. 32 (2017) 1093, https://doi.org/10.1080/09205071. 2017.1417919.

4. M. Eslami, Soliton-like solutions for the coupled SchrödingerBoussinesq equation, Optik 126 (2015) 3987. https:// doi.org/10.1016/j.ijleo.2015.07.197

5. D. J. Benney y A. C. Newell, The propagation of nonlinear wave envelopes, J. Math. Phys. 46 (1967) 133. https: //doi.org/10.1002/sapm1967461133

6. S. V. Manakov, On the theory of two-dimensional stationary self-focusing of electromagnetic waves, J. Exp. Theor. Phys. 38 (1974) 248.

7. Y. Kivshar y G. Agrawal, Optical solitons: from fibers to photonic crystals. (Academic Press, New York, 2003), https: //doi.org/10.1016/B978-0-12-410590-4. $\mathrm{X} 5000-1$

8. M. Asadzadeh, D. Rostamy y F. Zabihi, Discontinuous Galerkin and multiscale variational schemes for a coupled damped nonlinear system of Schrödinger equations, Numer. Methods Partial Differ. Equ. 29 (2013) 1912, https: / / doi. org/10.1002/num.21782

9. M. Delfour, M. Fortin y G. Payré, Finite-difference solutions of a non-linear Schrödinger equation, J. Comput. Phys. 44 (1981) 277, https://doi.org/10.1016/ 0021-9991 (81) 90052-8

10. Z. Fei, V. Pérez-García, y L. Vázquez, Numerical simulation of nonlinear Schrödinger systems: A new conservative scheme, Appl. Math. Comput. 71 (1995) 165, https://doi.org/ 10.1016/0096-3003(94)00152-T

11. Y.-F. Tang, L. Vázquez, F. Zhang y V. M. Pérez-García, Symplectic methods for the nonlinear Schrödinger equation, Comput. Math. Appl. 32 (1996) 73, https://doi.org/10. 1016/0898-1221(96)00136-8

12. S. López, J. Ochoa, y J. Gutiérrez, Exploring the behavior of solitons on a desktop personal computer, Rev. Mex. Fis. E 52 (2006) 28.

13. B. Moore y S. Reich, Backward error analysis for multisymplectic integration methods, Numer. Math. 95 (2003) 625. https://doi.org/10.1007/s00211-003-0458-9

14. M. Ismail y T. Taha, A linearly implicit conservative scheme for the coupled nonlinear Schrödinger equation, Math. Comput. Simul. 74 (2007) 302, https://doi.org/10.1016/ j.matcom.2006.10.020

15. B. Reichel y S. Leble, On convergence and stability of a numerical scheme of coupled nonlinear Schrödinger equations, Comput. Math. Appl. 55 (2008) 745. https://doi.org/ $10.1016 /$ j.camwa.2007.04.038
16. J. Cai, Multisymplectic schemes for strongly coupled Schrödinger system, Appl. Math. Comput. 216 (2010) 2417. https://doi.org/10.1016/j.amc.2010.03.087

17. Y. Wang y S. Li, New schemes for the coupled nonlinear Schrödinger equation, Int. J. Comput. Math. 87 (2010) 775. https://doi.org/10.1080/ $00207160802195985 \mathrm{~s}$.

18. M. S. Ismail y S. Z. Alamri, Higher accurate finite difference method for coupled nonlinear Schrödinger equation, Int. J. Comput. Math. 81 (2004) 333. https://doi.org/10. $1080 / 00207160410001661339$

19. M. S. Ismail, A fourth-order explicit schemes for the coupled nonlinear Schrödinger equation, Appl. Math. Comput., 196 (2008) 273. https://doi.org/10.1016/j.amc. 2007.05 .059

20. T. Wang, Optimal pointwise error estimate of a compact finite difference scheme for the coupled nonlinear schrödinger equations, J. Comput. Math. 32 (2014) 58. https://www. jstor.org/stable/43694041.

21. X. Hu y L. Zhang, Conservative compact difference schemes for the coupled nonlinear Schrödinger system, Numer. Methods Partial Differ. Equ., 30 (2014) 749. https : / / doi . org/ 10.1002 /num.21826

22. L. Kong, J. Hong, L. Ji, y P. Zhu, Compact and efficient conservative schemes for coupled nonlinear Schrödinger equations, Numer. Methods Partial Differ. Equ. 31 (2015) 1814. https : //doi.org/10.1002/num.21969

23. T. Bridges y S. Reich, Multi-symplectic integrators: numerical schemes for hamiltonian pdes that conserve symplecticity, Phys. Lett. A 284 (2001) 184. https://doi.org/10. 1016/S0375-9601(01)00294-8.

24. J.-Q. Sun y M.-Z. Qin, Multi-symplectic methods for the coupled 1d nonlinear Schrödinger system, Comput. Phys. Commun. 155 (2003) 221. https: / / doi.org/10.1016/ S0010-4655(03)00285-6

25. A. Aydin, Multisymplectic integration of n-coupled nonlinear Schrödinger equation with destabilized periodic wave solutions, Chaos Solitons Fractals, 41 (2009) 735. https:// doi.org/10.1016/j.chaos.2008.03.011

26. Y. Ma, L. Kong, J. Hong, y Y. Cao, High-order compact splitting multisymplectic method for the coupled nonlinear Schrödinger equations, Comput. Math. Appl. 61 (2011) 319. https://doi.org/10.1016/j.camwa. 2010.11 .007

27. Y. Chen, H. Zhu, y S. Song, Multi-symplectic splitting method for the coupled nonlinear Schrödinger equation, Comput. Phys. Commun. 181 (2010) 1231. https://doi.org/10. $1016 / j . c p c .2010 .03 .009$

28. X. Qian, S. Song, y Y. Chen, A semi-explicit multi-symplectic splitting scheme for a 3-coupled nonlinear schrödinger equation, Comput. Phys. Commun. 185 (2014) 1255. https:// doi.org/10.1016/j.cpc.2013.12.025 
29. M. Ismail y S. H. Alaseri, Computational methods for three coupled nonlinear Schrödinger equations, Appl. Math. 7 (2016) 2110. http://dx.doi.org/10.4236/am. 2016.717168

30. L. Kong, P. Wei, Y. Hong, P. Zhang, y P. Wang, Efficient energypreserving scheme of the three-coupled nonlinear Schrödinger equation, Math. Methods Appl. Sci. 42 (2019) 3222. https: //doi.org/10.1002/mma.5580

31. M. Song, X. Qian,, H. Zhang, J. Xia, y S. Song, Two kinds of new energy-preserving schemes for the coupled nonlinear Schrödinger equations, Commun. Comput. Phys. 25 (2019) 1127. https://doi.org/10.4208/cicp. OA-2017-0212

32. J. Cai, Y. Wang, y H. Liang, Local energy-preserving and momentum-preserving algorithms for coupled nonlinear Schrödinger system, J. Comput. Phys. 239 (2013) 30. https : //doi.org/10.1016/j.jcp.2012.12.036

33. P. Castillo y S. Gómez, Conservative local discontinuous Galerkin methods for a generalized system of strongly coupled nonlinear Schrödinger equations, Commun. Nonlinear Sci. Numer. Simul. 99 (2021) 105836. https://doi.org/10. $1016 / j . c n s n s .2021 .105836$

34. A. Aguilera, P. Castillo, y S. Gómez, Structure preservingField directional splitting difference methods for nonlinear Schrödinger systems, Appl. Math. Lett. 119 (2021) 107211. https://doi.org/10.1016/j.aml.2021.107211

35. P. Castillo y S. A. Gómez, Conservación de invariantes de la ecuación de Schrödinger no lineal por el método LDG, Rev. Mex. Fis. E. 64 (2018) 52. https://doi.org/10. 31349/RevMexFisE.64.52

36. S. K. Lele, Compact finite difference schemes with spectral-like resolution, J. Comput. Phys. 103 (1992) 16. https://doi. org/10.1016/0021-9991(92) 90324-R

37. P. Davis, Circulant matrices. (AMS Chelsea Publishing, New York, 1994).

38. W. Strauss y L. Vazquez, Numerical solution of a nonlinear Klein-Gordon equation, J. Comput. Phys. 28 (1978) 271. https://doi.org/10.1016/0021-9991(78) 90038-4

39. J. M. Sanza-Serna, Methods for the numerical solution of the nonlinear Schrödinger equation, Math.
Comp. 43 (1984) 21. https://doi.org/10.1090/ S0025-5718-1984-0744922-X

40. P. Castillo y S. Gómez, On the conservation of fractional nonlinear Schrödinger equation's invariants by the local discontinuous Galerkin method, J. Sci. Comput. 77 (2018) 1444. https: //doi.org/10.1007/s10915-018-0708-8

41. P. Castillo y S. Gómez, Conservative local discontinuous galerkin method for the fractional Klein-Gordon-Schrödinger system with generalized Yukawa interaction, Numer. Algorithms 84 (2020) 407. https://doi.org/10.1007/ s11075-019-00761-3

42. E. Hairer, C. Lubich, y G. Wanner, Geometric Numerical Integration (Springer, Berlin-Heidelberg, 2006), https : //doi. org/10.1007/3-540-30666-8

43. H. Yoshida, Construction of higher order symplectic integrators, Phys. Lett. A, 150 (1990) 262. https://doi.org/ $10.1016 / 0375-9601(90) 90092-3$

44. M. Suzuki, Fractal decomposition of exponential operators with applications to many-body theories and Monte Carlo simulations, Phys. Lett. A 146 (1990) 319. https://doi.org/ $10.1016 / 0375-9601(90) 90962-\mathrm{N}$

45. R. I. McLachlan, On the numerical integration of ordinary differential equations by symmetric composition methods, SIAM J. Sci. Comput. 16 (1995) 151, https://doi.org/10 . $1137 / 0916010$

46. W. Kahan y R.-C. Li, Composition constants for raising the orders of unconventional schemes for ordinary differential equations, Math. Comp. 66 (1997) 1089. https://doi.org/ 10.1090/S0025-5718-97-00873-9

47. R. I. McLachlan, Families of high-order composition methods, Num. Algorithms 31 (2002) 233, https://doi.org/10. 1023/A:1021195019574

48. R. M. Caplan y R. Carretero-González, Numerical stability of explicit Runge-Kutta finite-difference schemes for the nonlinear Schrödinger equation, Appl. Numer. Math., 71 (2013) 24, https://doi.org/10.1016/j.apnum. 2013.04 .002

49. D. E. Pelinovsky y J. Yang, Instabilities of multihump vector solitons in coupled nonlinear Schrödinger equations, Stud. Appl. Math. 115 (2008) 109, https://doi.org/10.1111/ j.1467-9590.2005.01565 